\title{
Understanding nuclear fuel forms and structures
}

\author{
David Frazer $^{1} \cdot$ Peter Hosemann ${ }^{2}$. Daniel Jädernäs ${ }^{3}$ · Erich Stergar ${ }^{4}$
}

Published online: 27 January 2022

(c) Idaho National Laboratory, under exclusive licence to The Materials Research Society 2022

Nuclear fuels experience some of the harshest operating conditions which greatly influence their microstructure evolution during use. It is necessary to understand the property and microstructure changes of fuel in order to evaluate their end-of-life behavior which is critical for the safe operation of a nuclear reactor. Evaluating these materials requires the use of novel materials characterization techniques such as advanced electron microscopy, small-scale mechanical testing, and localized pump probe experiments, allowing for novel insights into nuclear fuel systems. In this special issue, several of these advanced techniques are used to provide a wealth of new information on nuclear fuels.

The article by K.E. Wright et al. entitled "EPMA-based mass balance method for quantitative fission product distribution comparison between TRISO particles" presents work using the electron probe microanalysis to gain insight into the variability of burnup of particles in the same compact. The mass balance method employed compares well with ORIGEN-based fission product mass predictions.

J. Hiekinheimo et al. investigate "Iodine release from high-burnup fuel structures-Separate-effect tests and simulated fuel pellets for better understanding of iodine behaviour in nuclear fuels." The authors examine the release of iodine during annealing experiments using porous fuel surrogate samples. The results are then compared with fuel performance codes. Release behavior was influenced by the pellet sinter temperature, and the behavior may be dominated by other than simple grain boundary diffusion-related phenomena.

The article by J. Pakarinen et al. features proton irradiation work $\mathrm{UO}_{2}$ entitled "Proton irradiation-induced blistering in $\mathrm{UO}_{2}$." Proton irradiation was performed at $600{ }^{\circ} \mathrm{C}$

\footnotetext{
David Frazer

David.Frazer@inl.gov

Idaho National Laboratory, Idaho Falls, USA

University of California-Berkeley, Berkeley, USA

Studsvik AB, Nyköping, Sweden

SCK CEN, Mol, Belgium
}

with 3 different fluxes. Flaking of the $\mathrm{UO}_{2}$ occurred as twodimensional cavities were formed in the stopping peak.

The article entitled "Phase identification and morphology in rolled and annealed U-22.5at.\%Zr foils" by W.J. Williams et al. features the use of advanced electron microscopy techniques to quantify the phase fraction of $\delta-\mathrm{UZr}_{2}, \alpha-\mathrm{U}$, and inclusions. In the rolled sample, the phase fraction of $\delta-\mathrm{UZr}_{2}$ was significantly above the near equilibrium values observed in the annealed sample. The found results suggest a significant impact of the defect concentration on microstructural evolution and $\mathrm{Zr}$ migration within the fuel.

In the work by N.L. Cordes et al. entitled "Seeing through nuclear fuel: Three-dimensional, nondestructive X-ray microscopy and volumetric analyses of neutron-irradiated TRISO-coated fuel kernels," nondestructive X-ray tomography is used to make a 3D re-construction of a TRISO particle. The techniques applied enable volumetric analysis of UCO fuel kernels and voids in reasonable imaging times. This technique could be applied to irradiated fuel in the future.

Lastly, the work entitled "Nano-mechanical property assessment of a neutron irradiated HT-9 steel cladding and a fuel-cladding chemical interaction region of a uranium-10 wt\% zirconium nuclear fuel" by M. Okuniewski et al. combines nanoindentation and electron microscopy to thoroughly characterize microstructural and mechanical alterations in a highly irradiated HT-9 cladding. Three different regions across the thickness of the cladding could be identified and characterized. The results are also compared to literature data of un-irradiated and neutron-irradiated HT-9. 